\section{P. Dev}

Stanford University School of Medicine SUMMIT

Stanford, California, USA

\title{
Review
}

\section{Trends in Health Care Education: Research Opportunities in Teaching and Learning}

early exposure to clinical experience, and to link basic science research to clinical research and learning.

Together these forces are creating a new learning environment that supports active learning. Although these changes may take some decades to be completed, the learning tools and methods are already in use, curricula are being modified, and learning spaces are being remodeled to support the expected new learning methods. This process of change provides fertile ground for research on new curricula, new methods of content delivery, evaluation of their learning efficacy, and evaluation of the integration of change into the existing curriculum.
We discuss below some of the research opportunities that have arisen as a result of these changes.

\section{The student of the future}

The entering medical student of 2010 is in high school in the United States today and in even earlier grades in Europe and other countries. This student is accustomed to multitasking during work. While studying, she has music in the background, multipleinstant messaging screens on the computer monitor, or a study group in which she is participating. Current didactic, lecture-oriented teaching does not match the learning style to which this student is exposed. care education is the desire to increase

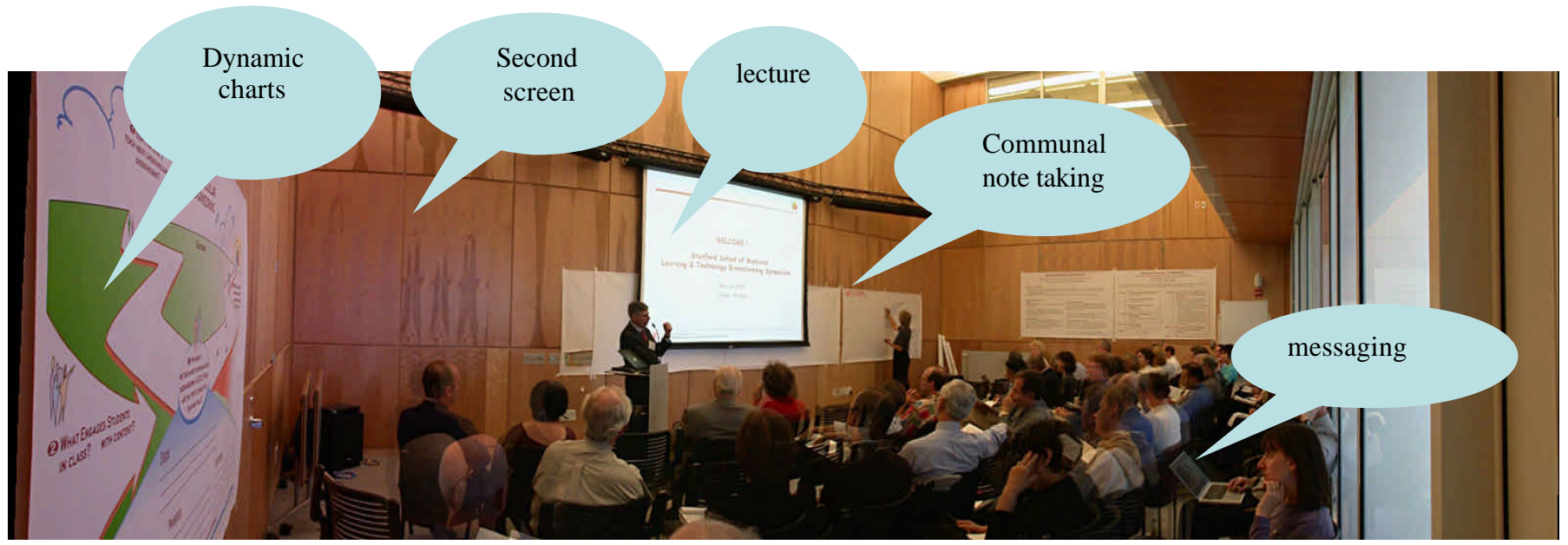

Fig. 1. A hypothetical evolution of the lecture. The lecture on the center screen is augmented by additional content (such as linked web sites) on the second screen. A screen on one side displays dynamic content, such as charts or views of a database, that evolve based on student activity within the classroom. The chart might show content such as the traversal of a decision tree as the entire group reasons through a clinical scenario. On the far right, a participant collects communal notes. In a digital classroom, a shared whiteboard would allow any student to transmit their notes to the whiteboard. Instant messaging and web surfing will be a natural part of class activity, supporting communication and acquisition of external information. 
Figure 1 suggests the type of large classroom interaction in which such a student may be immersed. Although a lecture often serves as a centerpiece or initiator of a learning session, there may be numerous other channels of information and communication. Related web sites may be shown on the second screen. Group or individual activity by the students may change a database, which is visualized as a dynamic chart. For example, ongoing opinion polls in the room can alter a decision tree visible on the chart. Discussion results and notes may be transmitted to an interactive whiteboard for communal note taking. In class laptops or hand-held devices may be used as a communication "back channel" for messaging between students. These devices may also be used for web surfing along with the lecture, adding richness and depth to the in-class discussion. This scenario of classroom activity highlights the many new ways in which students, teachers and content interaction will occur. In the past, the teacher's focus has been on excellent delivery of content through the lecture. Going forward, we expect that the focus will shift to the student's interaction with the content, and the teacher's interaction with the student.

\section{New resources for learning}

Digital learning resources include web sites, online courses and curricula, learning management systems, virtual patients, simulations of clinical and surgical procedures, and simulations of scenarios that represent multiperson interactions. We will highlight a few of these resources to indicate areas of change and topics of research.

\section{Online curricula}

Some schools have placed the content of the entire curriculum (including lectures, slides, handouts, quizzes and images) online on the World Wide Web. At Stanford, this transition has been gradual but marked; certain courses have seen a reduction in classroom attendance. That the system is well used is indicated by the complaints when the server or the network malfunctions. The impact of such access on classroom behavior, class attendance and study habits will be an interesting area of research for some years.

Professional groups with distributed clientele can benefit from having a uniform curriculum available at all sites and to all students, mentors and facilitators. A nutrition curriculum is illustrated in figure $2 a$. Figure $2 b$ shows an image from curriculum content for radiation dosimetrists who are being trained in the design of radiation treatment plans. Mentors at all locations can assign the online curriculum to their trainees, allowing formal education while providing practical training (apprenticeship) that is customized to their local resources. The curriculum content for radiation dosimetry is authored by a distributed group of faculty from twenty institutions, and production of this curriculum is a challenge in organizational behavior and motivation.

\section{Media libraries}

A bottleneck for the production of digital learning content has been the need to locate pertinent images and other media. The Slice of Life project was an early collection of pathology, anatomy and other images that found widespread use in the development of learning content around the world (Stensaas, 1994). At our institution, the MediaServer project has a goal of capturing specialty collections developed by our faculty, with annotations describing the content and its educational use (Duracket al, 2002). The multi-institution HEAL project has as agoal the development of a standardized metadata format so that multiple separate databases may be linked and searched (Candler et al, 2003).

The Visible Human male and female image datasets constitute a very large

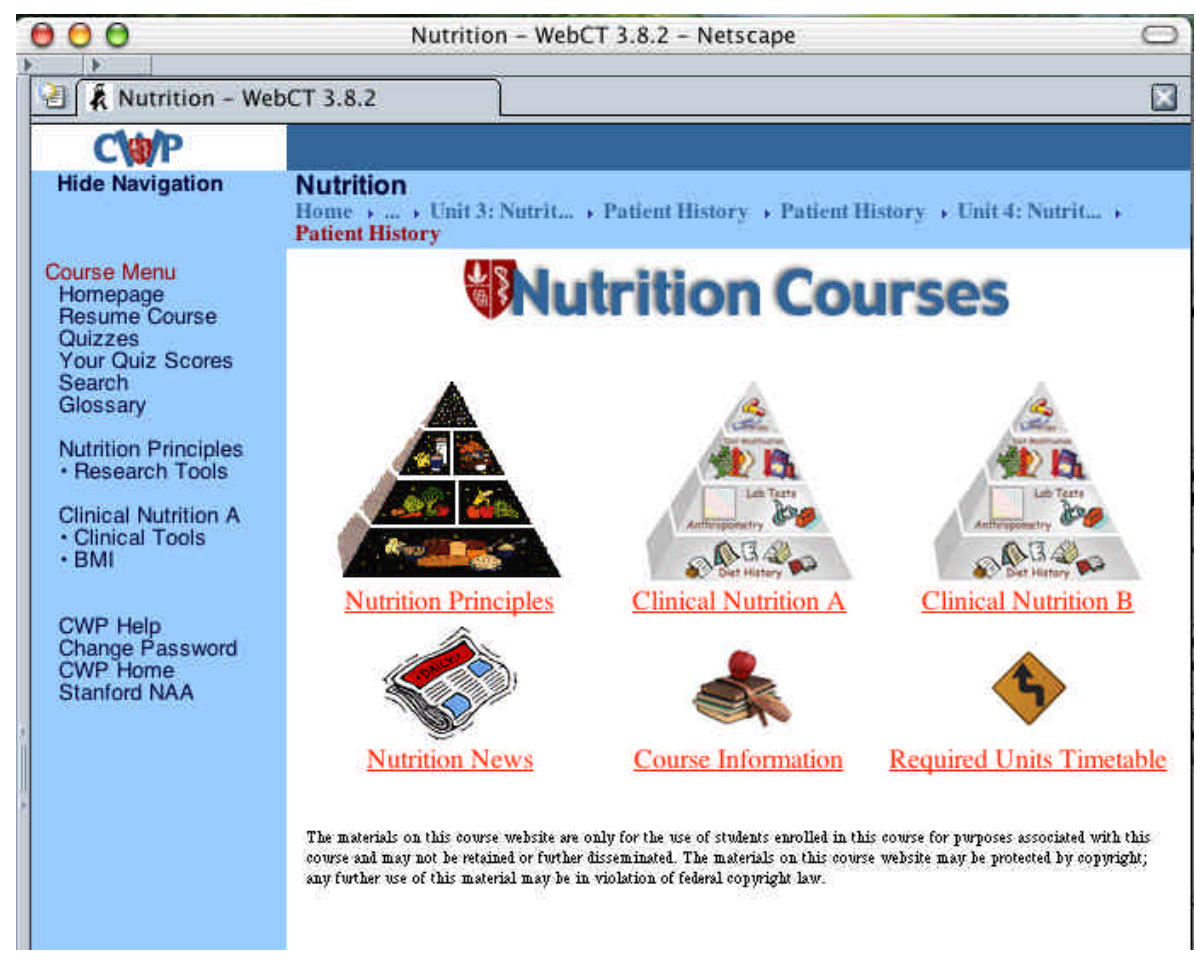

Fig. 2a. A nutrition curriculum for distance learning developed at Stanford. 


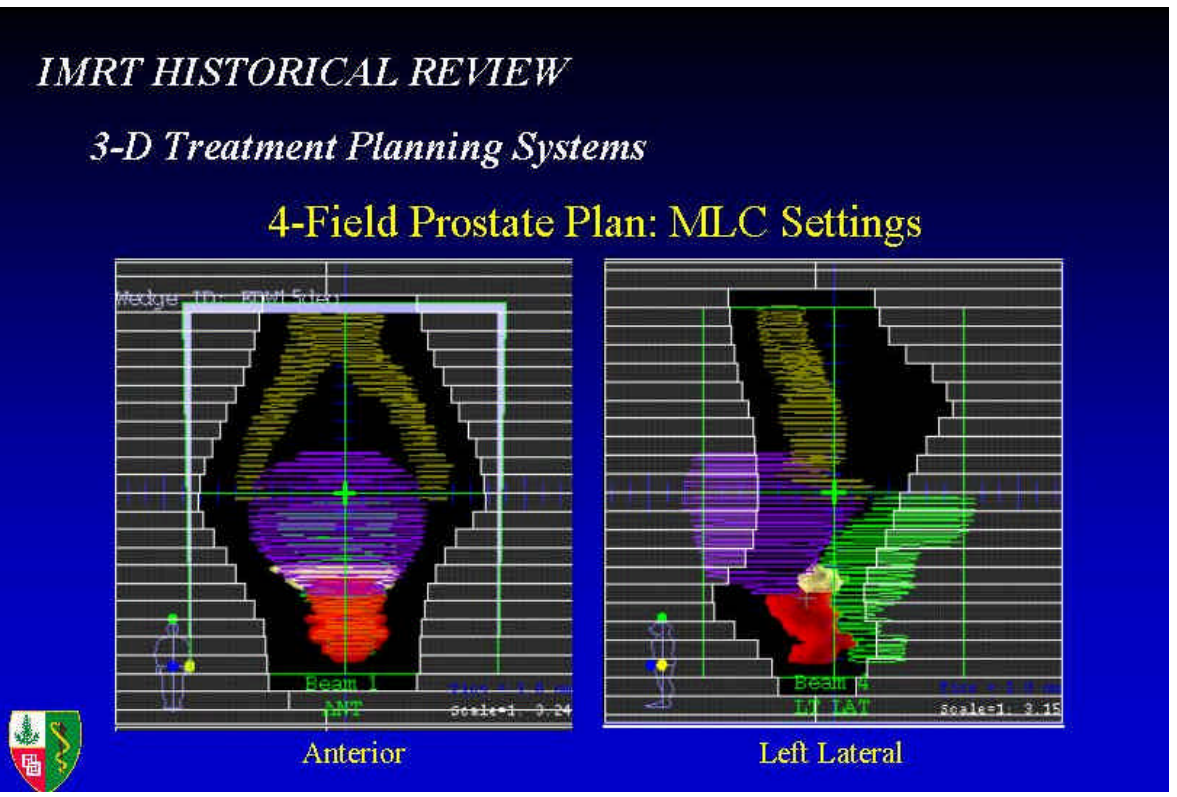

Fig. 2b. Media enriches the learning experience in the distance learning curriculum for training professional dosimetrists (courtesy Arthur Boyer, Stanford University).

collection of anatomic images (Spitzer et al, 1996). These and other anatomic image collections are beginning to be used as core resources in courses on human anatomy. When image pairs are available for viewing in stereo, providing a three-dimensional view of anatomy, the educational value of the images increases. In spite of the large size of these image collections, the computational power of current servers and the high bandwidth of Internet2 support almost instantaneous access to these images from any location. We therefore have the potential to make the human body available to any student at any location, including on a handheld device in the classroom or on the ward (Figure 3).

Research in media libraries falls within the larger scope of research in digital libraries. The definition of useful metadata, specialty-specific annotation, and interoperability of databases are all open technical challenges. Computation and rendering of image crosssections and of three-dimensional views are of ongoing interest. Given the fact that much of the media requires metadata whose entry cannot be automated, it is especially important to develop intuitive authoring interfaces.

\section{Virtual patient simulations}

Simulations of virtual patients range from full room mannequin-based simulation of an operating room, desktop simulations of interactive patients, and simulations of patients by live actors. Simulators represent the real medico-surgical world but provide a safe place where students may experiment without harm to the real patient. When the simulated case or procedure may be reused multiple times, the simulator supports a new mode of teaching and learning that encourages individual exploration and mastery.

The USMLE (United States Medical Licensing Examination) clinical skills examination proposes to use interactions between standardized patients and examinees to simulate one-on-one personal encounters between physicians and patients in a clinical setting. A key research challenge is to develop a scoring system assessment tool that predicts the clinical reasoning ability of the examinee (Clauser et al, 2002).
At Stanford, desktop simulations of virtual patients are being used to introduce students to history taking and clinical reasoning, to study presentation of infectious diseases, to expose students to issues of clinical nutrition, and to present problems of complex reasoning in pharmacologic management. Patient simulation by live actors (or standardized patients) is part of the program for introduction to clinical medicine, as well as a requirementfor graduation. Mannequin-based simulation of operating room scenarios is used for skills training and for training in team leadership, crisis management and communication under difficult conditions (Gaba and DeAnda, 1988).

A library of simulated cases could provide comprehensive standardized exposure to a clinical or surgical curriculum, augmenting the opportunistic experiences currently available in today's hospitals. In fact, interesting

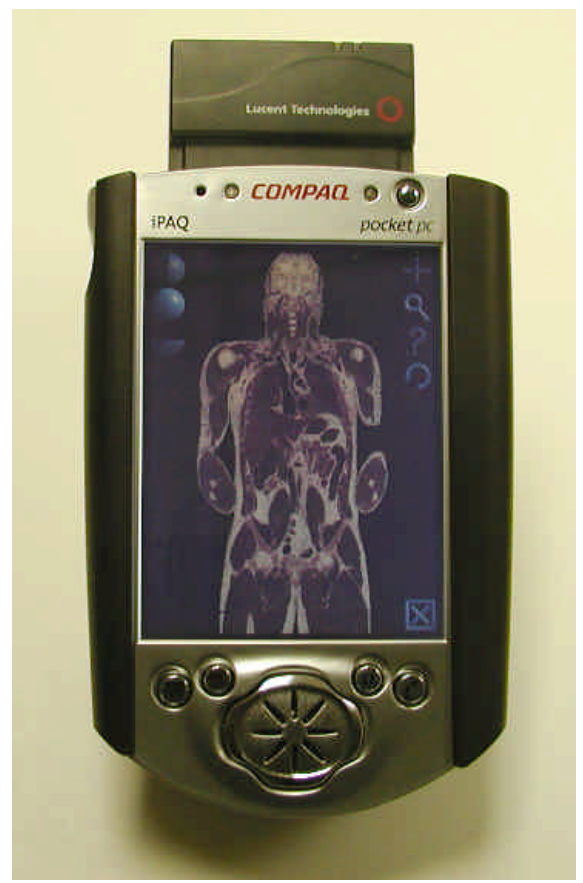

Fig. 3. The handheld device displays a slice through the Visible Human Male data. The data reside on a server at the University of Wisconsin, La Crosse, and can be accessed interactively by remote devices over the Internet. This image is computed by placing a cutting plane through a stack of horizontal cross-sectional images. 
clinical cases are often scarce, and a library of simulations can provide easy access to these valuable resources. In the near future, it is very possible that simulators will be used as tools for standardized assessment of clinical skills and reasoning.

\section{Clinical procedure simulators}

Procedure (or "part-task") simulators support the practice of clinical and surgical skills such as catheter or endoscope insertion, laparoscopic surgery and other tasks. These simulators usually provide a realistic tool as the interface device, such as the handle of a laparoscopic tool. In some cases, the tool has haptic or force feedback, allowing the user to sense touching or incising virtual anatomic parts (Figure 4). The simulators use computed graphics of virtual anatomy to help the learner visualize the procedure, such as the path of the needle or the wide-angle view through a laparoscopic camera. Besides providing visualization of the procedure, the display can facilitate collaborative learning, allowing discussion and experimentation (Hariri et al, in press).

The following are some examples of procedure simulators in routine curricular use. The e-Pelvis tool, designed to teach the examination of the female pelvis, makes the palpation actions of the student visible on a computer screen. This changes the learning process dramatically, making the examination of an internal cavity visible to the student, the teacher and to other students. A randomized control study demonstrated that students using the simulator performed better when tested on a standardized patient (live actor) than students who learned on a foam model or from books and videos (Pugh et al, 2001). The CathSim, a simulation of catheter insertion in a vein, provides haptic feedback (force feedback) as the needle penetrates the wall of the virtual vein. A construct

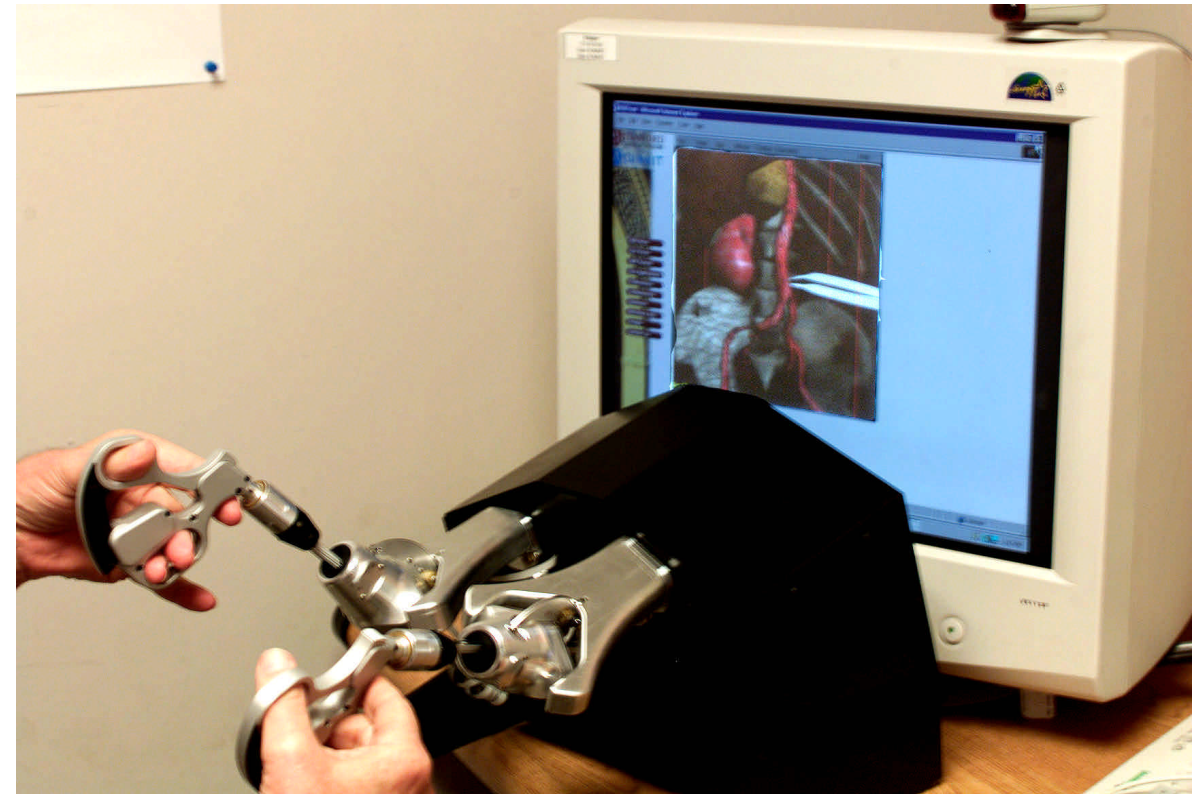

Fig. 4. A simulator for laparoscopic surgery. The learner manipulates the tools to act upon the virtual anatomy visible on the screen. As tissue is touched, pushed or cut, the learner feels the resistance through the tool handles.

validity study showed that experts performed better than novices, thus validating the simulator as a being a good representation of the process of intravenous catheter insertion (Reznek et al, 2002). CathSim is currently being used in a course on clinical procedures in emergency medicine.

Even though some are being sold commercially, procedure simulators are in their infancy, and research problems abound. The computer graphics and biomechanics computation for tissue and tool rendering raise problems of large scale and real-time computation and the modeling of physical systems (Dev et al, 2002). Haptic feedback modeling is based on finite element models or on representation of a mesh of springs and masses, and much refinement is possible. Making simulators available over networks requires addressing problems of network bandwidth and latency.Evaluation studies are required of simulator usability, validity, effectiveness as a learning tool, methods for integration in curriculum and the potential for transfer of learning to clinical and surgical practice.

\section{Evaluation}

Evaluation of learning tools is necessary before a new tool can be introduced into the curriculum. This should be done whether one acquires a completed tool or whether it is being developed locally. We have developed the SUMMIT seven-point framework that guides our evaluation of learning tools that are being considered for curricular use.

1. Reliability. Does the tool function as specified?

2. Usability. Is the tool easy to learn and to use?

3. Content. Has the content been reviewed for accuracy and appropriateness for the target audience?

4. Validity. Does the expert in the field perform better than the novice? Does a learner perform consistently during multiple uses of the tool?

5. Learning efficacy. Does learning occur when the tool is used? Is it the desired learning?

6. Curricular integration. Has the tool been integrated in curriculum? Is there a path to curricular integration? 
7. Change of practice. Does learning with the tool transfer to learning in the actual situation? Does the learner change how he practices once he uses the tool?

Every tool cannot be tested on all seven points. However, some tests, such as Reliability, are usually necessary before valid data can be obtained from subsequent tests.

\section{Supporting technology-rich learning environments}

Technology-rich environments require systems support, user support and support for development and use of learning resources (Figure 5).

Users include faculty, students and the staff that support the learning resources. User needs can vary widely, and a scenario-based approach can be useful in identifying user needs. We have used descriptive scenarios such as "A day in the life of a student / teacher / instructional designer" to capture the wide range of user needs. An ethnographic approach, where a staff member is shadowed and all interactions and requests captured, can detect user needs well beyond those elicited by interviews with users. These methods show that a user may have technical problems (i.e., difficulties with wireless connectivity), informational problems, (such as a need to collect clinical examples to teach a lecture on pharmacology), production problems (such as the best resolution at which to scan a radiograph), and pedagogic problems (such as the best instructional design for a thirty minute learning module on membrane proteins). The design of user support systems is a potentially fruitful research area that links work in education, workflow methods, organizational behavior, and technology.

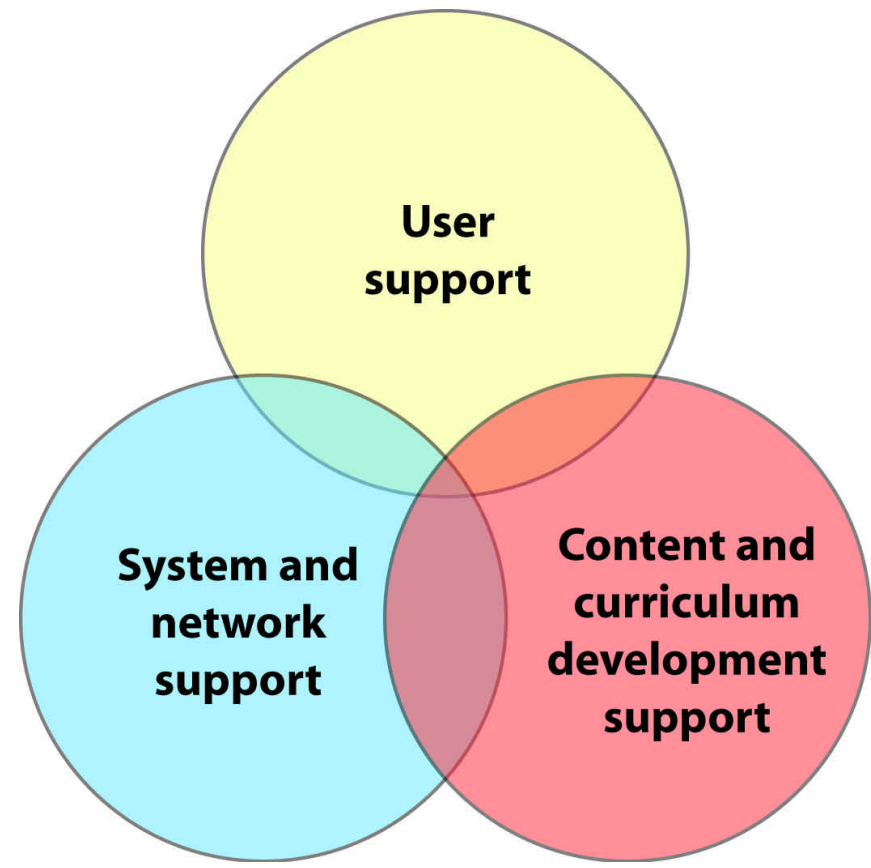

Fig. 5. The support infrastructure for learning technologies consists of three overlapping components.

Systems support includes support of desktop, server and networking equipment, specialized peripherals and the necessary system and software applications. System design that supports easy self-service is the first step in good support, since technologyrich classrooms, laboratories and discussion rooms are often used well beyond the typical eight-hour workday. User training and preventive maintenance are also integral to smooth technical operation. Specialized resources, such as simulators that include mannequin parts or surgical tools, or visualization systems with stereo display, will require additional technical support and extensive user training.

\section{Conclusion}

There are many forces changing health care education, and technology is central to the effectiveness of these changes. We have highlighted some of the resources that support these changes and have shown the range of research opportunities as these changes are implemented. Technology is expensive to develop and to implement. It is therefore imperative that we understand our educational goals and assess the potential impact of the changes before or during the process of change.

\section{References}

Barrows HS. A taxonomy of problem-based learning methods. Med Educ 1986 Nov;20(6):481-6.

Candler CS, Uijtdehaage SH, Dennis SE. Introducing HEAL: the Health Education Assets Library. Acad Med 2003 Mar;78(3):249-53.

Clauser BE, Margolis MJ, Swanson DB. An examination of the contribution of computer-based case simulations to the USMLE step 3 examination. Acad Med 2002 Oct;77(10 Suppl):S80-2.

Dev P, Montgomery K, Senger S, Heinrichs WL, Srivastava S, Waldron K. Simulated learning environments on the Internet. J Am Med Inform Assoc 2002 Sep-Oct; 9(5): 437-47.

Durack JC, Chao CC, Stevenson D, Andriole KP, Dev P. The Stanford MediaServer Project: Strategies for building a flexible digital media platform to support biomedical education and research. Proc AMIA Symp 2002. p.255-9. 
Gaba DM, DeAnda A. A comprehensive anesthesia simulation environment: recreating the operating room for research and training. Anesthesiology 1988 Sep;69(3):387-94.

Hariri S, Rawn C, Srivastava S, Youngblood P, Ladd A. Evaluation of a surgical simulator for learning clinical anatomy. MedEduc. In press 2003.

Pommert A, Hohne KH, Pflesser B, Richter E, Riemer M, Schiemann T, Schubert R, et al. Creating a high-resolution spatial/symbolic model of the inner organs based on the Visible Human. Med Image Anal 2001
Sep;5(3):221-8.

Pugh CM, Heinrichs WL, Dev P, Srivastava S, KrummelT. Objectiveassessment of clinical skills with a simulator, JAMA 2001 Sep 5;286(9):1021-3

Reznek MA, Rawn CL, Krummel TM. Evaluation of the educational effectiveness of a virtual reality intravenous insertion simulator. Acad Emerg Med 2002 Nov;9(11):1319-25.

Spitzer V, Ackerman MJ, Scherzinger AL, Whitlock D. The visible human male: a technical report. J Am Med Inform Assoc 1996 Mar-Apr;3(2):118-30.
Stensaas SS. Animating the curriculum: integrating multimedia into teaching. Bull Med Libr Assoc 1994 Apr;82(2):133-9.

Address of the author:

Parvati Dev

Stanford University School of Medicine SUMMIT

251 Campus Drive, MSOB - X226

Stanford, CA 94305-5466

USA

E-mail: dev@summit.stanford.edu 